\title{
Holography of massive M2-brane theory with discrete torsion
}

\author{
Dongmin Jang ${ }^{1, \mathrm{a}}$, Yoonbai Kim ${ }^{1, \mathrm{~b}}$, O-Kab Kwon ${ }^{1, \mathrm{c}}$, D. D. Tolla ${ }^{1,2, \mathrm{~d}}$ \\ ${ }^{1}$ Department of Physics, BK21 Physics Research Division, Autonomous Institute of Natural Science, Institute of Basic Science, \\ Sungkyunkwan University, Suwon 16419, Korea \\ ${ }^{2}$ University College, Sungkyunkwan University, Suwon 16419, Korea
}

Received: 5 September 2019 / Accepted: 2 February 2020 / Published online: 9 March 2020

(C) The Author(s) 2020

\begin{abstract}
We investigate the gauge/gravity duality between the $\mathcal{N}=6$ mass-deformed ABJ theory with $\mathrm{U}_{k}(N+l) \times$ $\mathrm{U}_{-k}(N)$ gauge symmetry and the 11-dimensional supergravity on LLM geometries with $\mathrm{SO}(2,1) \times \mathrm{SO}(4) / \mathbb{Z}_{k} \times$ $\mathrm{SO}(4) / \mathbb{Z}_{k}$ isometry and the discrete torsion $l$. For chiral primary operators with conformal dimensions $\Delta=1,2$, we obtain the exact vacuum expectation values using the holographic method in 11-dimensional supergravity and show that the results depend on the shapes of droplet pictures in LLM geometries. The $\frac{l}{\sqrt{N}}$ contributions from the discrete torsion $l$ for several simple droplet pictures in the large $N$ limit are determined in holographic vacuum expectation values. We also explore the effects of the orbifolding $\mathbb{Z}_{k}$ and the asymptotic discrete torsion $l$, on the gauge/gravity duality dictionary and on the nature of the asymptotic limits of the LLM geometries.
\end{abstract}

\section{Introduction}

The duality between the 3-dimensional $\mathcal{N}=6$ AharonyBergman-Jefferis-Maldacena (ABJM) theory with $\mathrm{U}_{k}(N) \times$ $\mathrm{U}_{-k}(N)$ gauge symmetry [1] and the M-theory on $\mathrm{AdS}_{4} \times$ $S^{7} / \mathbb{Z}_{k}$ geometry, together with the 4-dimensional $\mathcal{N}=4$ super Yang-Mills theory, which is dual to type IIB string theory on $\mathrm{AdS}_{5} \times S^{5}$ geometry [2,3], are thoroughly investigated cases of the gauge/gravity duality paradigm [2-5]. The non-conformal extension of the former, which is obtained by adding an appropriate mass deformation, preserves the full supersymmetry [6,7]. The mass-deformed ABJM (mABJM) theory is dual to the M-theory on 11-dimensional Lin-LuninMaldacena (LLM) geometries with $\mathrm{SO}(2,1) \times \mathrm{SO}(4) / \mathbb{Z}_{k} \times$

\footnotetext{
a e-mail: dongmin@skku.edu

be-mail: yoonbai@skku.edu

c e-mail: okab@skku.edu

de-mail: ddtolla@skku.edu (corresponding author)
}

$\mathrm{SO}(4) / \mathbb{Z}_{k}$ isometry $[8,9]$. Recently, we have made some meaningful tests of this duality [10-13]. We have calculated the vacuum expectation values (vevs) of chiral primary operators (CPOs) with conformal dimensions $\Delta=1$ and $\Delta=2$, from the classical vacuum solutions of mABJM theory and from the asymptotic expansion of the LLM solutions using the holographic methods. In the large $N$ limit, there is an agreement between the vevs obtained from the two sides. We have also calculated the entanglement entropy by using the Ryu-Takayanagi holographic formula [14] in the asymptotic limit of the LLM geometries and using the path integral methods developed in [15]. We have shown that the entanglement entropies obtained by using these two methods agree when the asymptotic limit of the LLM metric satisfies the linearized Einstein equation with an energy-momentum tensor for two scalar fields which encode the information of the mass deformation of the ABJM theory. See also Refs. [16-18] for related works.

An extension of the mABJM theory was achieved by widening the gauge symmetry as $\mathrm{U}_{k}(N+l) \times \mathrm{U}_{-k}(N)$, where $0 \leq l<k$ [19]. In the dual gravity theory, the $l$ was identified with the discrete torsion at asymptotic points of the LLM geometry with $\mathbb{Z}_{k}$ orbifold [9]. ${ }^{1}$ In general, the LLM geometries are represented by the droplet pictures, which are infinitely long strips with series of black/white regions along one space direction $\tilde{x}[8,9]$. In these droplet pictures, discrete torsions $\left\{l_{2 i+1}, l_{2 i}\right\}$ are assigned to the boundaries between the black/white regions and they count the number of fractional M2-branes at every odd boundary and fractional anti-M2-branes at every even boundary. In particular, the asymptotic discrete torsion $l$ belongs to the $\tilde{x}= \pm \infty$ points.

\footnotetext{
${ }^{1}$ In the ABJ theory [19], $l$ counts the number of fractional $M_{2}$ branes, whereas in the dual gravity theory, $l$ corresponds to the discrete torsion which is determined by the discrete holonomy of the 3 -form gauge field on a torsion 3-cycle $S^{3} / \mathbb{Z}_{k} \subset S^{7} / \mathbb{Z}_{k}$ i.e. $\int_{S^{3} / \mathbb{Z}_{k}} C_{3} \sim \frac{l}{k}$. See $[9,19]$ for details.
} 
The structure of the classical vacuum solutions in the extended mass-deformed Aharony-Bergman-Jefferis $(\mathrm{mABJ})$ theory is the same as that of the original $\mathrm{mABJM}$ theory, and are expressed in terms of the GRVV matrices [7]. Evaluation of the vevs from these classical vacuum solutions follows the same line as before. On the gravity side, we will argue that the LLM geometries with non-vanishing asymptotic discrete torsions are represented by droplet pictures where the origin is shifted by $l$ from the Fermi-level. As a result, we will show that the discrete torsions satisfy a modified level-matching condition and quantization condition of the 4-form flux. The holographic vevs are expressed as infinite summations involving these modified discrete torsions. In the general setting, converting these summations into expressions involving the parameters of the theory $(N, k, l)$ is out of our reach. Therefore, we rely on specific examples in order to determine the behaviour of the holographic vevs. In particular, our holographic vev for the CPO of conformal dimension $\Delta=1$ is given by

$\left\langle\mathcal{O}^{(\Delta=1)}\right\rangle_{\mathrm{HR}}=\frac{\mu_{0} N^{3 / 2}}{2 \pi} f_{0}(N, k, l)$,

where $\mu_{0}$ is the mass parameter of the LLM solutions, and we can exactly determine $f_{0}(N, k, l)$ which is finite in large $N$ limit.

On the other hand, the result obtained from the classical vacuum solutions on the field theory side is

$$
\begin{aligned}
\left\langle\mathcal{O}^{(\Delta=1)}\right\rangle_{\text {classical }}= & \frac{\mu_{0} N^{3 / 2}}{2 \pi}\left[f_{0}(N, k, l)+\frac{2 l}{\sqrt{N}}\right. \\
& \left.+\frac{f_{1}(k, l)}{N}+\cdots\right] .
\end{aligned}
$$

This shows that, for LLM geometries with non-vanishing asymptotic discrete torsion $l$, the first subleading term is $\mathcal{O}\left(\frac{1}{\sqrt{N}}\right)$, whereas it is $\mathcal{O}\left(\frac{1}{N}\right)$ when $l=0$. We will show that, in the special case of $k=1$, all the subleading terms are absent and there is an exact agreement between the results obtained from the classical vacuum solutions and from the holographic methods $[10,11]$. In general case of $k>1$, the gauge/gravity duality dictates that the vev obtained from the field theory calculations, including all quantum corrections, should agree with the holographic result. Therefore, we will argue that the subleading terms in (1.2) must be matched by the quantum corrections in order to get an agreement with (1.1). We will discuss the origin of the mismatch between Eqs. (1.1) and (1.2) in Sect. 4. The absence of the subleading terms in the $k=1$ case reveals some meaningful effect of the orbifold singularity on the nature of the gauge/gravity duality dictionary.

In order to obtain the holographic vev in (1.1), we need the asymptotic expansion of the LLM solutions. The asymptotic limit of LLM geometries represented by any droplet picture is the $\mathrm{AdS}_{4} \times S^{7} / \mathbb{Z}_{k}$ geometry. However, we will show that the radius of the asymptotic $S^{7}$ is given by

$L=\left(32 \pi^{2} A_{2}\right)^{1 / 6} l_{\mathrm{P}}$,

where $l_{\mathrm{P}}$ is the Planck length and $A_{2}$ is determined by the details of the droplet picture of the original LLM geometry. Therefore, even though every LLM geometry reduces asymptotically to the $\mathrm{AdS}_{4} \times S^{7} / \mathbb{Z}_{k}$ geometry, the fact that $L$ depends on $A_{2}$ suggests that there is some reminiscent information about the original LLM geometries in the asymptotic limit.

The remainder of this paper is organized as follows. In Sect. 2, we discuss the droplet representation of the LLM geometries with the asymptotic discrete torsion $l$ and obtain the appropriate level-matching and quantization conditions. We also introduce two quantities $\left(A_{2}\right.$ and $\left.A_{3}\right)$, and express them as infinite summations involving the discrete torsions. These two quantities are crucial to calculate the holographic vevs. In Sect. 3, based on our previous works, we summarize the Kaluza-Klein (KK) reduction of the 11-dimensional gravity on LLM geometries with $\mathbb{Z}_{k}$ orbifold to obtain 4-dimensional matter-gravity theory on $\mathrm{AdS}_{4}$ background geometry. In Sect. 4, we evaluate the vev of the CPO of conformal dimension $\Delta=1$ from the classical vacuum solutions of the mABJ theory. In Sect. 5, we calculate the holographic vev from the asymptotic expansion of the LLM solutions. We compare the holographic result with classical field theory result and identify the quantum corrections necessary for an agreement between the two results. For few simple droplet pictures in the large $N$ limit, the $\frac{l}{\sqrt{N}}$ contributions of the asymptotic discrete torsion $l$ are determined in holographic vev. We also comment on the holographic vev for CPO of $\Delta=2$. In Sect. 6, we draw our conclusions.

\section{LLM geometry with discrete torsion}

Some compelling evidences for the duality between the mABJM theory with $\mathrm{U}_{k}(N) \times \mathrm{U}_{-k}(N)$ gauge symmetry and the 11-dimensional supergravity on the LLM geometry with $\mathbb{Z}_{k}$ orbifold, are presented in [8-12]. It is also conjectured that the $\mathrm{mABJ}$ theory with $\mathrm{U}_{k}(N+l) \times \mathrm{U}_{-k}(N)$ gauge symmetry is dual to the LLM geometry with $\mathbb{Z}_{k}$ orbifold and asymptotic discrete torsion $l$. We begin this section by presenting a brief summary of the LLM geometry with asymptotic discrete torsion.

The metric and the corresponding 4-form field strength for the LLM geometry with $\mathbb{Z}_{k}$ orbifold and $\operatorname{SO}(2,1)$ $\times \mathrm{SO}(4) / \mathbb{Z}_{k} \times \mathrm{SO}(4) / \mathbb{Z}_{k}$ isometry, are given by [8]

$$
\begin{aligned}
d s^{2}= & -G_{t t}\left(-d t^{2}+d w_{1}^{2}+d w_{2}^{2}\right)+G_{x x}\left(d \tilde{x}^{2}+d \tilde{y}^{2}\right) \\
& +G_{\theta \theta} d s_{S^{3} / \mathbb{Z}_{k}}^{2}+G_{\tilde{\theta} \tilde{\theta}} d s_{\tilde{S}^{3} / \mathbb{Z}_{k}}^{2},
\end{aligned}
$$




$$
\begin{aligned}
F_{4}= & -d\left(e^{2 \Phi} h^{-2} V\right) \wedge d t \wedge d w_{1} \wedge d w_{2} \\
& +\mu_{0}^{-1}\left[V d\left(\tilde{y}^{2} e^{2 G}\right)+h^{2} e^{3 G} \star_{2} d\left(\tilde{y}^{2} e^{-2 G}\right)\right] \wedge d \Omega_{3} \\
& +\mu_{0}^{-1}\left[V d\left(\tilde{y}^{2} e^{-2 G}\right)-h^{2} e^{-3 G} \star_{2} d\left(\tilde{y}^{2} e^{2 G}\right)\right] \wedge d \tilde{\Omega}_{3},
\end{aligned}
$$

where $d s_{S^{3} / \mathbb{Z}_{k}}^{2}$ and $d s_{\tilde{S}^{3} / \mathbb{Z}_{k}}^{2}$ are metrics of two $S^{3}$ 's with $\mathbb{Z}_{k}$ orbifold while $d \Omega_{3}$ and $d \tilde{\Omega}_{3}$ are the corresponding volume forms $[9,20]$. The warp factors of the metric and the functions which determine the 4-form field strength $F_{4}$ are given by ${ }^{2}$

$G_{t t}=-\left(\frac{4 \mu_{0}^{2} \tilde{y} \sqrt{\frac{1}{4}-Z^{2}}}{f^{2}}\right)^{2 / 3}, \quad G_{x x}=\left(\frac{f \sqrt{\frac{1}{4}-Z^{2}}}{2 \mu_{0} \tilde{y}^{2}}\right)^{2 / 3}$,
$G_{\theta \theta}=\left(\frac{f \tilde{y} \sqrt{\frac{1}{2}+Z}}{2 \mu_{0}\left(\frac{1}{2}-Z\right)}\right)^{2 / 3}, \quad G_{\tilde{\theta} \tilde{\theta}}=\left(\frac{f \tilde{y} \sqrt{\frac{1}{2}-Z}}{2 \mu_{0}\left(\frac{1}{2}+Z\right)}\right)^{2 / 3}$,
$h^{2}=\frac{\sqrt{\frac{1}{4}-Z^{2}}}{\tilde{y}}, \quad e^{2 \Phi}=\frac{4 \tilde{y} \mu_{0}^{2} \sqrt{\frac{1}{4}-Z^{2}}}{f^{2}}, \quad e^{2 G}=\frac{\frac{1}{2}+Z}{\frac{1}{2}-Z}$

with

$f(\tilde{x}, \tilde{y})=\sqrt{1-4 Z^{2}-4 \tilde{y}^{2} V^{2}}$.

The geometry is completely determined by the two functions $Z(\tilde{x}, \tilde{y})$ and $V(\tilde{x}, \tilde{y})$,

$$
\begin{aligned}
& Z(\tilde{x}, \tilde{y})=\sum_{i=1}^{2 N_{b}+1} \frac{(-1)^{i+1}\left(\tilde{x}-\tilde{x}_{i}\right)}{2 \sqrt{\left(\tilde{x}-\tilde{x}_{i}\right)^{2}+\tilde{y}^{2}}}, \\
& V(\tilde{x}, \tilde{y})=\sum_{i=1}^{2 N_{b}+1} \frac{(-1)^{i+1}}{2 \sqrt{\left(\tilde{x}-\tilde{x}_{i}\right)^{2}+\tilde{y}^{2}}} .
\end{aligned}
$$

At $\tilde{y}=0$, the function $Z(\tilde{x}, \tilde{y})$ has values $\frac{1}{2}$ for $\tilde{x}_{2 i-1}<\tilde{x}<$ $\tilde{x}_{2 i}$ and $-\frac{1}{2}$ for $\tilde{x}_{2 i}<\tilde{x}<\tilde{x}_{2 i+1}$. Then the LLM geometries are represented by an infinite strip in the $\tilde{x}$-direction with regions of $Z(\tilde{x}, 0)=-\frac{1}{2}$ denoted by black color while regions of $Z(\tilde{x}, 0)=\frac{1}{2}$ denoted by white color and the $\tilde{x}_{i}$ 's are the positions of the boundaries between the black and white regions. See Fig. 1 below. The series of black/white regions are called droplets and each diagram contains $N_{b}$ black/white finite length droplets in addition to a semi-infinite black region below $\tilde{x}_{1}$ and a semi-infinite white region above $\tilde{x}_{2 N_{b}+1}$.

In any droplet picture, if one pulls down all the finite black regions to fill all the finite white regions, then the boundary

$\left.\overline{{ }^{2} \text { See }[10,11,13}\right]$ for detailed conventions.
Fig. 1 The droplet representation of the LLM geometries

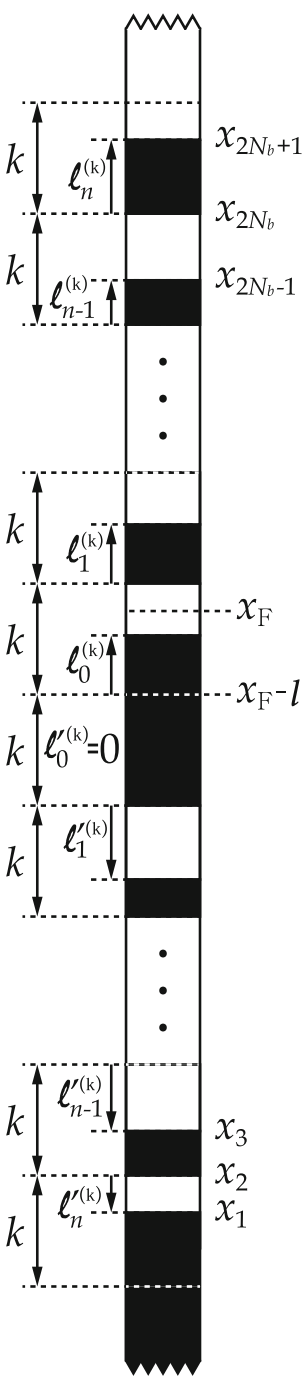

between the resulting semi-infinite black region and semiinfinite white region is located at

$\tilde{x}_{\mathrm{F}}=\tilde{x}_{1}+\sum_{i=1}^{N_{b}} \tilde{b}_{i}$,

where $\tilde{b}_{i}=\tilde{x}_{2 i+1}-\tilde{x}_{2 i}$ is the length of the $i$ th black region in the original diagram. This point is referred to as the Fermi level. In the original diagram, the total length of black regions above the Fermi level is equal to the total length of the white regions below the level.

In Ref. [9], it was proved that the 4-form flux through the $\mathbb{Z}_{k}$ orbifold of a 4-sphere that surrounds the $i$ th black/white region is proportional to the length of those regions, while the flux through the semi-infinite 4-cycle surrounding the semiinfinite black/white regions is proportional to the total lengths of all finite black/white regions. As a result, quantization of the fluxes leads to quantization of the lengths of those regions, namely 
$\tilde{x}_{i+1}-\tilde{x}_{i}=\frac{2 \pi l_{\mathrm{P}}^{3} \mu_{0}}{k} \mathbb{Z}$.

It was also verified that, introducing a rescaled coordinates $\mathrm{x}=\frac{\tilde{x}}{2 \pi l_{\mathrm{P}}^{3} \mu_{0}}$, the quantized lengths between $\mathrm{x}_{i}$ and $\mathrm{x}_{i+1}$ are related to the discrete torsions of the 3-form gauge field at those two boundaries as

$l_{i}+l_{i+1}= \pm\left(\mathrm{x}_{i+1}-\mathrm{x}_{i}\right) \bmod k$,

where the \pm signs are for the black/white regions and $l_{i}$ is the discrete torsion at $\mathrm{x}_{i}$. The recursion relation (2.11) is valid modulo $k$ since all the discrete torsions can be put in the range $0 \leq l_{i}<k$ by gauge transformation of the 3 -form gauge field.

The recursion relation (2.11) means all the discrete torsions can be fixed once we know the discrete torsions $\left(l_{0}=\right.$ $l_{2 N_{b}+2}=l$ ) of either at $\mathrm{x}= \pm \infty$, which are the discrete torsions of the asymptotic geometry $\mathrm{AdS}_{4} \times S^{7} / \mathbb{Z}_{k}$. It was argued that the discrete torsions at those asymptotic points are related to the rank of the $\mathrm{U}_{k}(N+l) \times \mathrm{U}_{-k}(N)$ gauge symmetry of the dual field theory $[9,19]$. For later convenience, we list the expressions for the discrete torsions at the odd and even boundary points as follows

$$
\begin{aligned}
l_{2 i+1} & =-\sum_{j=i+1}^{N_{b}} b_{i}+\sum_{j=1}^{i} w_{i}+l \bmod k \\
l_{2 i} & =\sum_{j=i}^{N_{b}} b_{i}-\sum_{j=1}^{i} w_{i}-l \bmod k,
\end{aligned}
$$

where $b_{i}=\mathrm{x}_{2 i+1}-\mathrm{x}_{2 i}$ and $w_{i}=\mathrm{x}_{2 i}-\mathrm{x}_{2 i-1}$ are the quantized lengths of the $i$ th black and white regions, respectively.

The discrete torsions $l_{2 i+1}$ are interpreted as fractional M2-branes at the odd fixed points $\mathrm{x}_{2 i+1}$, whereas the $l_{2 i}$ are interpreted as fractional anti-M2-branes at the even fixed points $\mathrm{x}_{2 i}$ [9]. However, it was illustrated that the solutions with anti-M2-branes can be geometrized to yield solutions with M2-branes only. Those solutions with M2-branes only are compared with vacuum solutions in the dual field theory. In this case the discrete torsions $l_{2 i}$ at the even fixed points are vanishing, i.e., the recursion relation (2.11) gives

$\mathrm{x}_{2 i+2}-\mathrm{x}_{2 i}=\mathbb{Z} k$.

This means in any droplet picture, the quantized distance between even fixed points is an integer multiple of $k$. Therefore, we can divide the droplet into intervals of length $k$ and all the even fixed points $\mathrm{x}_{2 i}$ coincide with the boundaries of some of the intervals. See Fig. 1.

\subsection{Shift of origin in the droplet picture}

Recalling $l_{2}=0$ and combining (2.12) and (2.9), we obtain

$\left(\mathrm{x}_{\mathrm{F}}-l\right)-\mathrm{x}_{2}=\mathbb{Z} k$.

Since $\mathrm{x}_{2}$ is at a boundary point of one of the intervals with length $k,(2.14)$ means that the point $\left(\mathrm{x}_{\mathrm{F}}-l\right)$ is also at one of those boundary points as in Fig. 1. For a non-vanishing $l$, we shift the origin of the droplet picture from the Fermi level $\mathrm{x}_{\mathrm{F}}$ to $\left(\mathrm{x}_{\mathrm{F}}-l\right)$. This shift of origin is required in order to get a correct level-matching condition. See below. The intervals both above and below the shifted origin are labelled by non-negative integers $n=0,1,2, \ldots$ starting at the origin. Any droplet picture is then parametrized by a set of integers $\left\{\ell_{n}^{(k)}, \ell_{n}^{\prime(k)}\right\}$, where $\ell_{n}^{(k)} / \ell_{n}^{\prime(k)}$ are the lengths of the black/white region in the $n$th interval above/below the origin. These integers are the discrete torsions assigned to the $n$th interval. Since the length of the black or white region in a given interval cannot be bigger than the length of the interval itself, these integers should satisfy the condition $0 \leq \ell_{n}^{(k)}, \ell_{n}^{\prime(k)} \leq k$.

For the $l=0$ case, counting of the intervals starts at the Fermi level, which implies the level-matching condition $\sum \ell_{n}^{(k)}=\sum \ell_{n}^{\prime(k)}$. However, in the case of non-vanishing $l$, the counting starts at $\left(\mathrm{x}_{\mathrm{F}}-l\right)$ point and then this shift of the origin increases the length of the black regions above or/and decreases the white regions below the origin. As a result, the level-matching condition is now given by

$\sum_{n=0}^{\infty} \ell_{n}^{(k)}=\sum_{n=0}^{\infty} \ell_{n}^{\prime(k)}+l$.

In addition, the relation between the discrete torsions and the number of M2-branes $N$ follows from the quantization of the 4-form flux [9],

$\sum_{n=0}^{\infty}\left(n+\frac{1}{2}\right)\left(\ell_{n}^{(k)}+\ell_{n}^{\prime(k)}\right)=N+\frac{l}{2}$.

\subsection{Invariant quantities under the shift of origin}

In the previous subsection, in order to obtain the correct levelmatching condition, we have shifted the origin of the droplet picture by $l$ below the Fermi level. For later convenience, here we discuss two important quantities which are invariant under such shift of the origin.

After some rearranging, the functions $Z(\tilde{x}, \tilde{y})$ and $V(\tilde{x}, \tilde{y})$ in (2.8) can be written in terms of the Legendre polynomials as follows [18], 


$$
\begin{aligned}
Z(r, \xi)= & \frac{1}{2}\left[\xi+\sum_{n=1}^{\infty} \mathrm{C}_{n}\left[(n+1) P_{n+1}(\xi)-2 \xi n P_{n}(\xi)\right.\right. \\
& \left.\left.+(n-1) P_{n-1}(\xi)\right]\left(\frac{2 \pi \mu_{0} l_{\mathrm{P}}^{3}}{r}\right)^{n}\right] \\
V(r, \xi)= & \frac{1}{2 r}\left[1+\sum_{n=1}^{\infty} \mathrm{C}_{n} P_{n}(\xi)\left(\frac{2 \pi \mu_{0} l_{\mathrm{P}}^{3}}{r}\right)^{n}\right],
\end{aligned}
$$

where $\xi=\frac{\tilde{x}}{r}$ with $r=\sqrt{\tilde{x}^{2}+\tilde{y}^{2}}$, and $P_{n}(\xi)$ are the Legendre polynomials. We have also introduced $[10,11]^{3}$

$\mathrm{C}_{n}=\sum_{i=1}^{2 N_{b}+1}(-1)^{i+1}\left(\frac{\tilde{x}_{i}}{2 \pi \mu_{0} l_{\mathrm{P}}^{3}}\right)^{n}$.

The two shift-invariant quantities of our interest are

$$
A_{2}=\frac{1}{2}\left(\mathrm{C}_{2}-\mathrm{C}_{1}^{2}\right), \quad A_{3}=\frac{1}{3}\left(\mathrm{C}_{3}-3 \mathrm{C}_{1} \mathrm{C}_{2}+2 \mathrm{C}_{1}^{3}\right) .
$$

These are shift invariants because they can be written in terms of shift invariant variables $b_{i}$ and $w_{i}$, which are the lengths of the black and white regions, as

$$
\begin{aligned}
A_{2} & =\sum_{i=1}^{N_{b}} \sum_{j=i}^{N_{b}} w_{i} b_{j}, \\
A_{3} & =\sum_{i=1}^{N_{b}}\left(\sum_{j=i}^{N_{b}} \sum_{k=1}^{j} w_{i} b_{j} w_{k}-\sum_{j=1}^{i} \sum_{k=j}^{N_{b}} b_{i} w_{j} b_{k}\right) .
\end{aligned}
$$

These invariant quantities will play central role in the asymptotic expansion of the LLM solutions and consequently in the holographic determination of the vevs of CPOs.

In $[10,11]$, we have obtained the expressions of those two invariant quantities in terms of the discrete torsions $\left\{\ell_{n}, \ell_{n}^{\prime}\right\}$ for the special case of $(k=1, l=0)$, where the discrete torsions $\left\{\ell_{n}, \ell_{n}^{\prime}\right\}$ are either 0 or 1 . The results were

$A_{2}=\sum_{n=0}^{\infty}\left(n+\frac{1}{2}\right)\left(\ell_{n}+\ell_{n}^{\prime}\right), \quad A_{3}=\sum_{n=0}^{\infty} n(n+1)\left(\ell_{n}-\ell_{n}^{\prime}\right)$.

We have also verified that, in this special case, $A_{2}=N$, with $N$ being the rank of the gauge group, and $A_{3}$ is proportional to the vev of a CPO of conformal dimension one in the dual field theory. Here, we follow some indirect procedure in order to obtain the expressions for $A_{2}$ and $A_{3}$ for the case of $(k>1$, $0 \leq l<k)$. To that end, let us rewrite (2.21) by shifting down

\footnotetext{
${ }^{3}$ Note that our definition for $\mathrm{C}_{n}$ here differs from that of $[10,11]$ by factor of $A^{-n / 2}$, where $A$ is area of Young diagram.
}

the origin of the droplet picture by $l$, so that the counting of discrete torsions starts from $\mathrm{x}_{\mathrm{F}}-l$. In order to account for this shift of the origin, we rename the discrete torsions as in the following table

\begin{tabular}{llll}
\hline$\left\{\ell_{n}, \ell_{n}^{\prime}\right\}, l=0$ & $\longrightarrow$ & $\left.\tilde{\ell}_{n}, \tilde{\ell}_{n}^{\prime}\right\}, l>0$ & range of $n$ \\
\hline \hline$\ell_{n-l}$ & $\longrightarrow$ & $\tilde{\ell}_{n}$ & $n \geq l$ \\
\hline $1-\ell_{l-1-n}^{\prime}$ & $\longrightarrow$ & $\tilde{\ell}_{n}$ & $0 \leq n \leq l-1$ \\
\hline$\ell_{n+l}^{\prime}$ & $\longrightarrow$ & $\tilde{\ell}_{n}^{\prime}$ & $n \geq 0$ \\
\hline
\end{tabular}

In terms of the renamed discrete torsions, the quantities in (2.21) are written as

$$
\begin{aligned}
& A_{2}=\sum_{n=0}^{\infty}\left(n+\frac{1}{2}\right)\left(\tilde{\ell}_{n}+\tilde{\ell}_{n}^{\prime}\right)-\frac{l^{2}}{2} \\
& A_{3}=\sum_{n=0}^{\infty} n(n+1)\left(\tilde{\ell}_{n}-\tilde{\ell}_{n}^{\prime}\right)-l\left(l^{2}-1\right)-2 A_{2} l .
\end{aligned}
$$

Next, we write (2.23) in terms of the discrete torsions for the case of $(k>1,0 \leq l<k)$. We note that, because of the constraints in (2.13) and (2.14), not all droplet pictures which are allowed in the $k=1$ case are allowed in the $k>1$. Any droplet picture with arbitrary lengths of the black/white regions is consistent with (2.13) and (2.14) in the $k=1$ case, whereas only some subset of those droplet pictures are consistent with those constraints in the $k>1$ case. For those droplet pictures in the subset, the discrete torsions $\left\{\ell_{n}^{(k)}, \ell_{n}^{\prime(k)}\right\}$ for $k>1$ can be written in terms of $\left\{\tilde{\ell}_{n}, \tilde{\ell}_{n}^{\prime}\right\}$ introduced in (2.22) as follows

$\ell_{m}^{(k)}=\sum_{n=k m}^{k(m+1)-1} \tilde{\ell}_{n}, \quad \ell_{m}^{\prime(k)}=\sum_{n=k m}^{k(m+1)-1} \tilde{\ell}_{n}^{\prime}$.

Rearranging (2.23) and using (2.24), one can rewrite it in terms of $\left\{\ell_{n}^{(k)}, \ell_{n}^{\prime(k)}\right\}$ as

$$
\begin{aligned}
A_{2}= & k\left[\sum_{n=0}^{\infty}\left(n+\frac{1}{2}\right)\left(\ell_{n}^{(k)}+\ell_{n}^{\prime(k)}\right)-\frac{l}{2}\right] \\
& -\frac{1}{2} \sum_{n=0}^{\infty}\left[\ell_{n}^{(k)}\left(k-\ell_{n}^{(k)}\right)+\ell_{n}^{\prime(k)}\left(k-\ell_{n}^{\prime(k)}\right)\right] \\
& +\frac{1}{2} l(k-l), \\
A_{3}= & k^{2} \sum_{n=0}^{\infty} n(n+1)\left(\ell_{n}^{(k)}-\ell_{n}^{\prime(k)}\right) \\
& -k \sum_{n=0}^{\infty} n\left[\ell_{n}^{(k)}\left(k-\ell_{n}^{(k)}\right)-\ell_{n}^{\prime(k)}\left(k-\ell_{n}^{\prime(k)}\right)\right] \\
& +\frac{1}{3} \sum_{n=0}^{\infty}\left(\ell_{n}^{(k) 3}-\ell_{n}^{\prime(k) 3}\right)-\frac{1}{3} l^{3}-2 A_{2} l .
\end{aligned}
$$


These results are crucial in subsequent sections for our holographic calculations of the vevs of $\mathrm{CPO}$ with conformal dimensions one and two.

\section{KK reduction}

In this section, we review the KK reduction of the 11dimensional supergravity to a 4-dimensional matter-gravity theory, where the matter content of the latter is determined by the LLM solutions. The KK reduction involves an expansion of the 11-dimensional fluctuations in terms of the spherical harmonics on $S^{7} / \mathbb{Z}_{k}$ and then projecting the equations of motions on those spherical harmonics to obtain the equations of motion for various KK modes. At non-linear order, the resulting equations contain higher derivative terms of the 11-dimensional fluctuations. Then one needs to introduce the KK maps to absorb the higher derivative terms and obtain the canonical equations of motion for the 4-dimensional modes. A detailed KK reduction procedure at non-linear order is carried out in [10-13]. Here, for clarity, we briefly summarize the procedure and write the equations of motion for a few 4-dimensional modes which are necessary for our purpose.

\subsection{Field equations at quadratic order}

In order to write the 11-dimensional gravity equations of motion up to quadratic order in the fluctuations, we perturb the 11-dimensional fields around the $\mathrm{AdS}_{4} \times S^{7} / \mathbb{Z}_{k}$ background as

$\mathbf{g}_{p q}=g_{p q}+h_{p q}, \quad \mathbf{F}_{p q r s}=F_{p q r s}+f_{p q r s}$,

where $p, q, \ldots=0, \ldots, 10$. The equations of motion for those fluctuations are obtained by inserting (3.26) into the 11-dimensional gravity equations of motion and keeping all the terms which are quadratic in the fluctuations $h_{p q}$ and $f_{\text {pqrs }}$. The resultant equations are

$$
\begin{aligned}
& \nabla^{r} \nabla_{p} h_{q r}+\nabla^{r} \nabla_{q} h_{p r}-\nabla^{2} h_{p q}-\nabla_{q} \nabla_{p} h_{r}^{r}-R h_{p q} \\
& \quad-g_{p q}\left(-R^{r s} h_{r s}+\nabla^{r} \nabla^{s} h_{r s}-\nabla^{2} h^{r}{ }_{r}\right) \\
& \quad+\frac{1}{48}\left(F_{r s t u} F^{r s t u} h_{p q}-4 g_{p q} h_{r s} F^{r}{ }_{t u v} F^{s t u v}\right) \\
& \quad+\frac{1}{24} g_{p q} f_{r s t u} F^{r s t u}-\frac{1}{2} h_{r s} F^{r}{ }_{p t u} F_{q}{ }^{s t u} \\
& \quad-\frac{1}{6}\left(f_{p r s t} F_{q}^{r s t}+F_{p r s t} f_{q}^{r s t}\right)=0 \\
& \nabla_{p}\left(h^{t}{ }_{t} F^{p q r s}\right)+2 \nabla_{p}\left(4 F_{t}{ }^{[p q r} h^{s] t}+f^{p q r s}\right) \\
& \quad+\frac{2}{\sqrt{-g}} \frac{1}{(4 !)^{2}} \tilde{\epsilon}^{p_{1} \ldots p_{4} q_{1} \ldots q 4 q r s} f_{p_{1} \ldots p_{4}} F_{q_{1} \ldots q_{4}}=0
\end{aligned}
$$

where the indices are raised/lowered by the $\operatorname{AdS}_{4} \times S^{7} / \mathbb{Z}_{k}$ background metric and the covariant derivatives as well as the Ricci tensor are also those of the background.

Next we expand the fluctuations in terms of the spherical harmonics on $S^{7} / \mathbb{Z}_{k}$, with $\mathrm{SO}(4) / \mathbb{Z}_{k} \times \mathrm{SO}(4) / \mathbb{Z}_{k}$ symmetry, so that the orbifolding does not affect the expansion,

$h_{\mu \nu}(x, y)=h_{\mu \nu}^{I_{1}}(x) Y^{I_{1}}(y), \quad h_{\rho}^{\rho}(x, y)=h^{I_{1}}(x) Y^{I_{1}}(y)$,

$h_{(a b)}=s^{I_{1}}(x) \nabla_{(a} \nabla_{b)} Y^{I_{1}}(y), \quad h_{a}^{a}(x, y)=\phi^{I_{1}}(x) Y^{I_{1}}(y)$,

$f_{\mu \nu \rho \sigma}(x, y)=\frac{2}{3} \nabla_{[\mu} t^{\lambda I_{1}}(x) \epsilon_{\nu \rho \sigma] \lambda} Y^{I_{1}}(y)$,

$f_{\mu \nu \rho a}(x, y)=-\frac{1}{3 !} \epsilon_{\mu \nu \rho}{ }^{\sigma} t_{\sigma}^{I_{1}}(x) \nabla_{a} Y^{I_{1}}(y)$,

$f_{\mu a b c}(x, y)=\nabla_{\mu} t^{I_{35}}(x) Y_{a b c}^{I_{35}}(y)$,

$f_{a b c d}(x, y)=4 t^{I_{35}}(x) \nabla_{[a} Y_{b c d]}^{I_{35}}(y)$,

where $I_{n}=0,1,2, \ldots$. Here we have split the 11dimensional indices into the $\operatorname{AdS}_{4}$ indices $(\mu, v, \ldots=$ $0, \ldots, 3)$ and the $S^{7}$ indices $(a, b, \ldots=4, \ldots, 10), x$ denotes the $\mathrm{AdS}_{4}$ coordinates, whereas $y$ denotes the $S^{7}$ coordinates, and $\left(Y^{I_{1}}, Y_{a b c}^{I_{35}}\right)$ are the scalar and antisymmetric 3-tensor spherical harmonics on $S^{7}$, respectively.

Plugging (3.29) into (3.27) and (3.28), we obtain the equations for various modes. The details can be found in [13]. Here we copy the equations for the graviton mode $\hat{h}_{\mu \nu}^{0}$ and two scalar modes $\Psi$ and $T$

$$
\begin{aligned}
& \left(L_{E}+\frac{12}{L^{2}}\right) \hat{h}_{\mu \nu}^{0} \\
& +\frac{1}{34560}\left\{-\frac{26}{3} \nabla_{\mu} \Psi \nabla_{\nu} \Psi\right. \\
& +\frac{28}{3} \Psi \nabla_{\mu} \nabla_{\nu} \Psi+\frac{L^{2}}{3} \nabla_{\mu} \nabla^{\rho} \Psi \nabla_{\nu} \nabla_{\rho} \Psi \\
& +\frac{L^{2}}{2} \nabla^{\rho} \Psi \nabla_{\mu} \nabla_{\nu} \nabla_{\rho} \Psi+\frac{L^{4}}{24} \nabla_{\mu} \nabla^{\rho} \nabla^{\sigma} \Psi \nabla_{\nu} \nabla_{\rho} \nabla_{\sigma} \Psi \\
& +\frac{L^{4}}{32} \nabla^{\rho} \nabla^{\sigma} \Psi \nabla_{\mu} \nabla_{\nu} \nabla_{\rho} \nabla_{\sigma} \Psi \\
& -g_{\mu \nu}\left(\frac{12}{L^{2}} \Psi^{2}+\nabla^{\rho} \Psi \nabla_{\rho} \Psi+\frac{35 L^{2}}{48} \nabla^{\rho} \nabla^{\sigma} \Psi \nabla_{\rho} \nabla_{\sigma} \Psi\right. \\
& \left.\left.\quad-\frac{L^{4}}{64} \nabla^{\tau} \nabla^{\rho} \nabla^{\sigma} \Psi \nabla_{\tau} \nabla_{\rho} \nabla_{\sigma} \Psi\right)\right\} \\
& +\frac{1}{48}\left(T \nabla_{\mu} \nabla_{\nu} T+\frac{1}{2} \nabla_{\mu} T \nabla_{\nu} T\right) \\
& +\frac{1}{96} g_{\mu \nu}\left(\nabla_{\rho} T \nabla^{\rho} T-\frac{16}{L^{2}} T^{2}\right)=0, \\
& \left(\square+\frac{8}{L^{2}}\right) \Psi+\mathcal{O}\left(\mu_{0}^{3}\right)=0, \quad\left(\square+\frac{8}{L^{2}}\right) T+\mathcal{O}\left(\mu_{0}^{3}\right)=0,
\end{aligned}
$$


where $L_{E}$ represents the Einstein operator, and we have introduced the following diagonal modes

$\hat{h}_{\mu \nu}^{0} \equiv h_{\mu \nu}^{0}-\frac{1}{4} g_{\mu \nu} \phi^{0}+\frac{1}{24} g_{\mu \nu} \hat{\psi}^{0}$,

$\Psi \equiv \frac{1}{70}\left(7 \hat{\psi}^{2}-162 \hat{\phi}^{2}\right), \quad T \equiv t^{I_{35}=1}$

with $\hat{\phi}^{I_{1}} \equiv \phi^{I_{1}}+\frac{I_{1}\left(I_{1}+6\right)}{L^{2}} s^{I_{1}}, \quad \hat{\psi}^{I_{1}} \equiv 18 h^{I_{1}}-L \nabla^{\mu} t_{\mu}^{I_{1}}$.

We use the following field redefinition in order to absorb the higher derivative terms in the graviton equation (3.30), and obtain the canonical 4-dimensional graviton mode

$$
\begin{aligned}
H_{\mu \nu}= & \hat{h}_{\mu \nu}^{0}+g_{\mu \nu}\left(C_{1} \Psi^{2}+C_{2} \nabla^{\rho} \Psi \nabla_{\rho} \Psi\right)+C_{3} \nabla_{\mu} \Psi \nabla_{\nu} \Psi \\
& +g_{\mu \nu} C_{4} \nabla^{\rho} \nabla^{\sigma} \Psi \nabla_{\rho} \nabla_{\sigma} \Psi \\
& +C_{5} \nabla_{\mu} \nabla^{\rho} \Psi \nabla_{\nu} \nabla_{\rho} \Psi+g_{\mu \nu} C_{T} T^{2}
\end{aligned}
$$

where

$$
\begin{aligned}
& C_{1}=-\frac{1}{2^{6} 3^{3} 5}, \quad C_{2}=-\frac{L^{2}}{2^{11} 3^{3} 5}, \\
& C_{3}=-\frac{7 L^{2}}{2^{11} 3^{4} 5}, C_{4}=-\frac{L^{4}}{2^{14} 3^{3} 5}, \\
& C_{5}=-\frac{L^{4}}{2^{13} 3^{4} 5}, \quad C_{T}=-\frac{1}{2^{5} 3} .
\end{aligned}
$$

Consequently the graviton equation (3.30) becomes

$$
\begin{gathered}
\left(L_{E}+\frac{12}{L^{2}}\right) H_{\mu \nu}-\frac{1}{96}\left(\nabla_{\mu} T \nabla_{\nu} T+\frac{M_{t}^{2}}{2} g_{\mu \nu} T^{2}\right) \\
-\frac{1}{2304}\left(\nabla_{\mu} \Psi \nabla_{\nu} \Psi+\frac{M_{\psi}^{2}}{2} g_{\mu \nu} \Psi^{2}\right)=0 .
\end{gathered}
$$

\subsection{Asymptotic expansion of LLM geometries}

The $\frac{1}{r}$ expansion of the functions $Z(r, \xi)$ and $V(r, \xi)$ to all orders are given in (2.17), however it is difficult to obtain such closed form for the expansions of the functionals in (2.6). Therefore, for the warp factors in (2.6), we write the asymptotic expansion by keeping only the leading and subleading terms,

$$
\begin{aligned}
G_{t t}(r, \xi)= & -\frac{r^{2}}{2^{2 / 3} \pi^{4 / 3} l_{\mathrm{P}}^{4}\left(\mathrm{C}_{2}-\mathrm{C}_{1}^{2}\right)^{2 / 3}} \\
& \times\left(1-\frac{2^{2} \pi l_{\mathrm{P}}^{3}\left(2 \mathrm{C}_{3}-3 \mathrm{C}_{2} \mathrm{C}_{1}+\mathrm{C}_{1}^{3}\right)}{3\left(\mathrm{C}_{2}-\mathrm{C}_{1}^{2}\right) r} \mu_{0} \xi+\cdots\right), \\
G_{r r}(r, \xi)= & \frac{\pi^{2 / 3} l_{\mathrm{P}}^{2}\left(\mathrm{C}_{2}-\mathrm{C}_{1}^{2}\right)^{1 / 3}}{2^{2 / 3} r^{2}} \\
& \times\left(1+\frac{2^{2} \pi l_{\mathrm{P}}^{3}\left(\mathrm{C}_{3}-\mathrm{C}_{1}^{3}\right)}{3\left(\mathrm{C}_{2}-\mathrm{C}_{1}^{2}\right) r} \mu_{0} \xi+\cdots\right), \\
G_{\theta \theta}(r, \xi)= & 2^{1 / 3} \pi^{2 / 3} l_{\mathrm{P}}^{2}\left(\mathrm{C}_{2}-\mathrm{C}_{1}^{2}\right)^{1 / 3}(1+\xi)
\end{aligned}
$$

$$
\begin{aligned}
\times & \left(1+\frac{2 \pi l_{\mathrm{P}}^{3}}{3\left(\mathrm{C}_{2}-\mathrm{C}_{1}^{2}\right) r} \mu_{0}\left[\left(2 \mathrm{C}_{3}-3 \mathrm{C}_{2} \mathrm{C}_{1}+\mathrm{C}_{1}^{3}\right) \xi\right.\right. \\
& \left.\left.-3\left(\mathrm{C}_{2} \mathrm{C}_{1}-\mathrm{C}_{1}^{3}\right)\right]+\cdots\right), \\
G_{\tilde{\theta} \tilde{\theta}}(r, \xi)= & -2^{1 / 3} \pi^{2 / 3} l_{\mathrm{P}}^{2}\left(\mathrm{C}_{2}-\mathrm{C}_{1}^{2}\right)^{1 / 3}(1-\xi) \\
& \times\left(1-\frac{2 \pi l_{\mathrm{P}}^{3}}{3\left(\mathrm{C}_{2}-\mathrm{C}_{1}^{2}\right) r} \mu_{0}\left[\left(2 \mathrm{C}_{3}-3 \mathrm{C}_{2} \mathrm{C}_{1}+\mathrm{C}_{1}^{3}\right) \xi\right.\right. \\
& \left.\left.+3\left(\mathrm{C}_{2} \mathrm{C}_{1}-\mathrm{C}_{1}^{3}\right)\right]+\cdots\right),
\end{aligned}
$$

where we made the replacement $G_{r r}(r, \xi) \equiv G_{x x}(\tilde{x}, \tilde{y} \rightarrow$ $r, \xi)$.

By recalling that the asymptotic limit of the LLM geometry is the $\mathrm{AdS}_{4} \times S^{7} / \mathbb{Z}_{k}$, the leading terms of the above warp factors should give those of the $\mathrm{AdS}_{4} \times S^{7} / \mathbb{Z}_{k}$ geometry. In particular, we should have

$\lim _{r \rightarrow \infty} G_{r r}(r, \xi)=\frac{L^{2}}{4 r^{2}}$,

where $L$ is the radius of the $S^{7}$. Comparing (3.37) with the expansion of $G_{r r}(r, \xi)$ in (3.36), we obtain

$L=\left(32 \pi^{2} A_{2}\right)^{1 / 6} l_{\mathrm{P}}$,

where we have used the definition of $A_{2}$ in (2.19). With the result in (3.38), we reproduce the $\operatorname{AdS}_{4} \times S^{7} / \mathbb{Z}_{k}$ metric by plugging the leading terms of the warp factors in (3.36) into the LLM metric in (2.4).

A comment is in order about the result in (3.38). In the previous section, we have discussed the LLM geometries which are classified by their droplet representations. For every droplet picture, we have the corresponding set of the discrete torsions $\left\{\ell_{n}^{(k)}, \ell_{n}^{\prime(k)}\right\}$, which are constrained by the level-matching condition (2.15) and the quantization condition (2.16). The asymptotic limit of all these LLM geometries is the $\mathrm{AdS}_{4} \times S^{7} / \mathbb{Z}_{k}$ geometry. However, since the radius $L$ in (3.38) depends on $A_{2}$, which is expressed in terms of the discrete torsions as in (2.25), there is some reminiscent information about the original LLM geometries in the asymptotic $\mathrm{AdS}_{4} \times S^{7} / \mathbb{Z}_{k}$ geometry.

Now using the coordinate transformation

$\rho=\frac{L^{3}}{4 r}$,

we rewrite (3.36) as

$$
\begin{aligned}
G_{t t}(\rho, \xi)= & \frac{L^{2}}{2^{2 / 3}} \frac{1}{4 \rho^{2}} \\
& \times\left(1-\frac{2^{4} \pi l_{\mathrm{P}}^{3}\left(2 C_{3}-3 C_{2} C_{1}+C_{1}^{3}\right)}{3 L^{3}\left(C_{2}-C_{1}^{2}\right)} \mu_{0} \rho \xi+\cdots\right),
\end{aligned}
$$




$$
\begin{aligned}
G_{\rho \rho}(\rho, \xi)= & \frac{L^{2}}{4 \rho^{2}}\left(1+\frac{2^{4} \pi l_{\mathrm{P}}^{3}\left(C_{3}-C_{1}^{3}\right)}{3 L^{3}\left(C_{2}-C_{1}^{2}\right)} \mu_{0} \rho \xi+\cdots\right), \\
G_{\theta \theta}(\rho, \xi)= & \frac{L^{2}}{2}(1+\xi) \\
& \times\left(1+\frac{2^{3} \pi l_{\mathrm{P}}^{3}}{3 L^{3}\left(C_{2}-C_{1}^{2}\right)} \mu_{0} \rho\left[\left(2 C_{3}-3 C_{2} C_{1}+C_{1}^{3}\right) \xi\right.\right. \\
& \left.\left.-3 C_{1}\left(C_{2}-C_{1}^{2}\right)\right]+\cdots\right), \\
G_{\tilde{\theta} \tilde{\theta}}(\rho, \xi)= & \frac{L^{2}}{2}(1-\xi) \\
& \times\left(1+\frac{2^{3} \pi l_{\mathrm{P}}^{3}}{3 L^{3}\left(C_{2}-C_{1}^{2}\right)} \mu_{0} \rho\left[\left(2 C_{3}-3 C_{2} C_{1}+C_{1}^{3}\right) \xi\right.\right. \\
& \left.\left.+3 C_{1}\left(C_{2}-C_{1}^{2}\right)\right]+\cdots\right) .
\end{aligned}
$$

In order to read the vevs of gauge invariant operators from the asymptotic expansions of gravity solutions, the FeffermanGraham (FG) coordinates system is the most convenient. In the FG coordinates, the LLM metric (2.4) is written as

$$
\begin{aligned}
d s^{2}= & \frac{L^{2}}{4 z^{2}}\left(d z^{2}+\frac{4 z^{2}}{L^{2}} G_{t t}(z, \tau)\left(-d t^{2}+d w_{1}^{2}+d w_{2}^{2}\right)\right) \\
& +G_{\tau \tau}(z, \tau) d \tau^{2}+G_{\theta \theta}(z, \tau) d s_{S^{3} / \mathbb{Z}_{k}}^{2} \\
& +G_{\tilde{\theta} \tilde{\theta}}(z, \tau) d s_{\tilde{S}^{3} / \mathbb{Z}_{k}}^{2} .
\end{aligned}
$$

The warp factors in (3.36) are written in the FG coordinates as

$$
\begin{aligned}
G_{t t}(z, \tau) & =\frac{L^{2}}{4 z^{2}}-\frac{A_{3} L^{2} \mu_{0}}{2 \sqrt{2} A_{2}^{3 / 2} z}+\mathcal{O}\left(\mu_{0}^{2}\right), \\
G_{\tau \tau}(z, \tau) & =\frac{L^{2}}{4\left(1-\tau^{2}\right)}+\mathcal{O}\left(\mu_{0}^{2}\right), \\
G_{\theta \theta}(z, \tau) & =\frac{L^{2}}{2}(1+\tau)+\frac{A_{3} L^{2} \mu_{0} z}{2 \sqrt{2} A_{2}^{3 / 2}}(1+\tau)^{2}+\mathcal{O}\left(\mu_{0}^{2}\right), \\
G_{\tilde{\theta} \tilde{\theta}}(z, \tau) & =\frac{L^{2}}{2}(1-\tau)+\frac{A_{3} L^{2} \mu_{0} z}{2 \sqrt{2} A_{2}^{3 / 2}}(1-\tau)^{2}+\mathcal{O}\left(\mu_{0}^{2}\right),
\end{aligned}
$$

where we have used the following coordinate transformation

$$
\begin{aligned}
& \rho(z, \tau)=z+\frac{2\left(C_{1}^{3}-C_{3}\right) \mu_{0} z^{2}}{3\left(C_{2}-C_{1}^{2}\right)^{3 / 2}} \tau+\mathcal{O}\left(\mu_{0}^{2}\right), \\
& \xi(z, \tau)=\tau+\frac{2\left(C_{1}^{3}-C_{3}\right) \mu_{0} z}{3\left(C_{2}-C_{1}^{2}\right)^{3 / 2}}\left(\tau^{2}-1\right)+\mathcal{O}\left(\mu_{0}^{2}\right) .
\end{aligned}
$$

Similarly, the asymptotic expansions of the 4-form field strength of the LLM solution in (2.5) are given by

$$
\begin{aligned}
& F_{t w_{1} w_{2} z}(z, \tau)=-\frac{3 L^{3}}{8 z^{4}}+\frac{3 A_{3} L^{3} \mu_{0}}{2^{5 / 2} A_{2}^{3 / 2} z^{3}} \tau+\mathcal{O}\left(\mu_{0}^{2}\right), \\
& F_{t w_{1} w_{2} \tau}(z, \tau)=-\frac{3 A_{3} L^{3} \mu_{0}}{2^{7 / 2} A_{2}^{3 / 2} z^{2}}+\mathcal{O}\left(\mu_{0}^{2}\right), \\
& F_{\theta \phi \psi z}(z, \tau)=-\frac{1}{8} L^{3} \mu_{0} \sin \theta(1+\tau)^{2}+\mathcal{O}\left(\mu_{0}^{2}\right), \\
& F_{\theta \phi \psi \tau}(z, \tau)=-\frac{1}{4} L^{3} \mu_{0} z \sin \theta(1+\tau)+\mathcal{O}\left(\mu_{0}^{2}\right), \\
& F_{\tilde{\theta} \tilde{\phi} \tilde{\psi} z}(z, \tau)=-\frac{1}{8} L^{3} \mu_{0} \sin \tilde{\theta}(1-\tau)^{2}+\mathcal{O}\left(\mu_{0}^{2}\right), \\
& F_{\tilde{\theta} \tilde{\phi} \tilde{\psi} \tau}(z, \tau)=\frac{1}{4} L^{3} \mu_{0} z \sin \tilde{\theta}(1-\tau)+\mathcal{O}\left(\mu_{0}^{2}\right) .
\end{aligned}
$$

The expansion in (3.42) shows that in the asymptotic region, the warp factors for the LLM metric are given by the $\mathrm{AdS}_{4} \times S^{7} / \mathbb{Z}_{k}$ background and small fluctuations. Therefore, in the asymptotic region, the LLM metric are written as in (3.26), where the values of the fluctuations $h_{p q}$ are read from the asymptotic expansion in (3.42). Similarly, from the asymptotic expansion of the 4-form field strength in (3.44), we read the values of the fluctuations $f_{p q r s}$. Finally, the obtained values of the fluctuations $h_{p q}$ and $f_{\text {pqrs }}$ can be expanded in terms of the spherical harmonics on $S^{7} / \mathbb{Z}_{k}$ as in (3.29), in order to obtain the values of the KK modes $\left(h_{\mu \nu}^{I_{1}}, s^{I_{1}}, \phi^{I_{1}}, \ldots\right)$. Here, we are interested only in three physical modes which consist of the graviton mode $H_{\mu \nu}$ and the two scalar modes, $\Psi$ and $T$, introduced in the previous subsection. From (3.32) and (3.33), we notice that these three physical modes are composed of KK modes $\left(h_{\mu \nu}^{0}, s^{2}, \phi^{2}, \ldots\right)$. Therefore, we obtain the values of the physical modes by combining the values of the KK modes, which in turn are read from the asymptotic expansion of the LLM geometry. ${ }^{4}$ See Refs. $[10,11]$ for the detailed procedure. The results are

$$
\begin{aligned}
H_{i j} & =\left[-\frac{\left(L \mu_{0}\right)^{2}}{180}\left(30+\beta_{3}^{2}\right)+\mathcal{O}\left(\mu_{0}^{4}\right)\right] \eta_{i j}, \\
H_{z z} & =-\frac{\left(L \mu_{0}\right)^{2}}{1440}\left(960+29 \beta_{3}^{2}\right)+\mathcal{O}\left(\mu_{0}^{4}\right), \\
\Psi & =-24 \beta_{3} \mu_{0} z+\mathcal{O}\left(\mu_{0}^{3}\right), \quad T=16 \sqrt{3} \mu_{0} z+\mathcal{O}\left(\mu_{0}^{3}\right),
\end{aligned}
$$

where $\eta_{i j}=\operatorname{diag}(-1,1,1)$ and

$\beta_{3}=\frac{3 A_{3}}{A_{2}^{3 / 2}}$.

\footnotetext{
${ }^{4}$ Similar procedure was developed in $[21,22]$ to investigate the holographic dual of the Coulomb branch in $\mathcal{N}=4$ Yang-Mills theory.
} 


\section{Vacuum solutions of mass-deformed ABJ theory and vevs of CPOs}

The mass deformation of the $\mathcal{N}=6$ ABJM theory with $\mathrm{U}_{k}(N) \times \mathrm{U}_{-k}(N)$ gauge group preserves full supersymmetry but breaks $\mathrm{SU}(4)$ global R-symmetry to $\mathrm{SU}(2) \times \mathrm{SU}(2) \times$ $\mathrm{U}(1)$. When $l$ overlapping fractional M2-branes are added at $\mathbb{Z}_{k}$ orbifold fixed points, the gauge group of the theory is extended to $\mathrm{U}_{k}(N+l) \times \mathrm{U}_{-k}(N)$ with $0 \leq l<k$. This theory is often called the ABJ theory [19]. The vacuum solutions in the mass-deformed ABJ theory are composed of the GRVV matrices, which are also the building blocks of the vacuum solutions in the mass-deformed ABJM theory.

In order to reflect the $\mathrm{SU}(2) \times \mathrm{SU}(2) \times \mathrm{U}(1)$ global symmetry, the four complex scalars $\left(Y^{A}, A=1,2,3,4\right)$ are split into two two-component scalars as

$Y^{A}=\left(Z^{a}, W^{\dagger a}\right), \quad a=1,2$.

Then, the vacuum solutions are,

$$
\begin{aligned}
Z_{0}^{a} & =\sqrt{\frac{k \mu}{2 \pi}} \oplus_{n=0}^{\infty}\left(\left[\begin{array}{cc}
{\left[M_{(n)}^{a}\right]_{n \times(n+1)} \otimes I_{N_{n}}} & 0_{n \times n} \\
0_{n+1 \times n+1} & 0_{(n+1) \times n} \otimes I_{N_{n}^{\prime}}
\end{array}\right),\right. \\
W_{0}^{\dagger a} & =\sqrt{\frac{k \mu}{2 \pi}} \oplus_{n=0}^{\infty}\left(\begin{array}{cc}
0_{n \times(n+1)} \otimes I_{N_{n}} & 0_{n \times n} \\
0_{n+1 \times n+1} & {\left[\bar{M}_{(n)}^{a}\right]_{(n+1) \times n} \otimes I_{N_{n}^{\prime}}}
\end{array}\right),
\end{aligned}
$$

where $\mu=4 \mu_{0}$ and $M_{(n)}^{a}$ are the irreducible GRVV matrices,

$M_{(n)}^{1}=\left(\begin{array}{cccccc}\sqrt{n} & 0 & & & & \\ & \sqrt{n+1} & 0 & & & \\ & & \ddots & \ddots & & \\ & & & \sqrt{2} & 0 & \\ & & & & 1 & 0\end{array}\right)$,

$M_{(n)}^{2}=\left(\begin{array}{cccccc}0 & 1 & & & & \\ & 0 & \sqrt{2} & & & \\ & & \ddots & \ddots & & \\ & & & 0 & \sqrt{n+1} & \\ & & & & 0 & \sqrt{n}\end{array}\right)$

with $\bar{M}_{(n)}^{a}=\left(M_{(n)}^{a}\right)^{\dagger}$. Each vacuum solution contains $N_{n}$ irreducible matrices of the first type $M_{(n)}^{a}$ and $N_{n}^{\prime}$ of the second type $\bar{M}_{(n)}^{a}$. The set of integers $\left\{N_{n}, N_{n}^{\prime}\right\}$ are called occupation numbers. Since $\left(Z^{a}, W^{\dagger a}\right)$ are $(N+l) \times N$ matrices, the occupation numbers should satisfy the relations,

$\sum_{n=0}^{\infty}\left(n+\frac{1}{2}\right)\left(N_{n}+N_{n}^{\prime}\right)=N+\frac{l}{2}, \quad \sum_{n=0}^{\infty}\left(N_{n}-N_{n}^{\prime}\right)=l$.
The vacuum solutions are supesymmetric for $0 \leq N_{n}, N_{n}^{\prime} \leq$ $k$ [23]. There is one-to-one correspondence between the LLM solutions and the vacuum solutions of the mABJM theory [9]. This correspondence is realized through a one-to-one map between the discrete torsions and the occupation numbers,

$\left\{\ell_{n}^{(k)}, \ell_{n}^{\prime(k)}\right\} \Longleftrightarrow\left\{N_{n}, N_{n}^{\prime}\right\}$

The CPO of conformal dimension one, which is invariant under the $\mathrm{SU}(2) \times \mathrm{SU}(2) \times \mathrm{U}(1)$ global symmetry with nonvanishing vev, is given by $[10,11]$

$\mathcal{O}^{(\Delta=1)}=\frac{1}{4} \operatorname{Tr}\left(Z^{a} Z_{a}^{\dagger}-W^{\dagger a} W_{a}\right)$.

For later convenience, lets discuss the nature of the vev of such operators. Let a complex scalar fields $Y^{A}=\left(Z^{a}, W^{\dagger a}\right)$ and near the vacuum that can be written as $Y^{A}(x)=Y_{0}^{A}+$ $\delta Y^{A}(x)$, where $Y_{0}^{A}$ is the vacuum solutions represented by GRVV matrices [7]. Then the vev of a gauge invariant operator $\mathcal{O}=M_{A}{ }^{B} \operatorname{Tr}\left(Y^{A} Y_{B}^{\dagger}\right)$, with $M_{A}{ }^{B}=\operatorname{diag}(1,1,-1,-1)$ is given by

$$
\begin{aligned}
\langle\mathcal{O}\rangle_{\mathrm{mABJ}}= & \int \mathcal{D} Y e^{i\left(S_{0}+S_{\mu}\right)} M_{A}{ }^{B}\left(\operatorname{Tr}\left(Y_{0}^{A} Y_{B 0}^{\dagger}\right)+\operatorname{Tr}\left(Y_{0}^{A} \delta Y_{B}^{\dagger}\right)\right. \\
& \left.+\operatorname{Tr}\left(\delta Y^{A} Y_{B 0}^{\dagger}\right)+\operatorname{Tr}\left(\delta Y^{A} \delta Y_{B 0}^{\dagger}\right)\right),
\end{aligned}
$$

where $S_{0}$ is the action of the original conformal ABJM theory and

$S_{\mu}=\mu^{2} \int \operatorname{Tr}\left(Y Y^{\dagger}\right)+\frac{4 \pi \mu}{k} \int \operatorname{Tr}\left(Y Y^{\dagger} Y Y^{\dagger}\right)$

is the mass deformation. Here we have suppressed the indices of $Y$ for simplicity, see [6,7] for the exact forms of the quadratic and quartic mass deformations. Expanding (4.53) in powers of the mass parameter, one can write

$$
\begin{aligned}
\langle\mathcal{O}\rangle_{\mathrm{mABJ}}= & M_{A}{ }^{B}\left[\operatorname{Tr}\left(Y_{0}^{A} Y_{B 0}^{\dagger}\right)+\left\langle\operatorname{Tr}\left(Y_{0}^{A} \delta Y_{B}^{\dagger}\right)\right\rangle_{0}\right. \\
& +\left\langle\operatorname{Tr}\left(\delta Y^{A} Y_{B 0}^{\dagger}\right)\right\rangle_{0}+\left\langle\operatorname{Tr}\left(\delta Y^{A} \delta Y_{B 0}^{\dagger}\right)\right\rangle_{0} \\
& +\frac{4 \pi \mu}{k}\left(\operatorname{Tr}\left(Y_{0}^{A} Y_{B 0}^{\dagger}\right)\left\langle\int \operatorname{Tr}\left(Y Y^{\dagger} Y Y^{\dagger}\right)\right\rangle_{0}\right. \\
& +\left\langle\operatorname{Tr}\left(Y_{0}^{A} \delta Y_{B}^{\dagger}\right) \int \operatorname{Tr}\left(Y Y^{\dagger} Y Y^{\dagger}\right)\right\rangle_{0} \\
& +\left\langle\operatorname{Tr}\left(\delta Y^{A} Y_{B 0}^{\dagger}\right) \int \operatorname{Tr}\left(Y Y^{\dagger} Y Y^{\dagger}\right)\right\rangle_{0} \\
& \left.\left.+\left\langle\operatorname{Tr}\left(\delta Y^{A} \delta Y_{B 0}^{\dagger}\right) \int \operatorname{Tr}\left(Y Y^{\dagger} Y Y^{\dagger}\right)\right\rangle_{0}\right)\right]+\mathcal{O}\left(\mu^{2}\right),
\end{aligned}
$$

where $\langle\cdots\rangle_{0}$ denote $n$-point functions in the conformal ABJM theory. Noting that the one-point functions in the first 
line are vanishing in the conformal ABJM theory and the multi-trace contributions from the second line onwards are $\frac{1}{N}$-suppressed as compared to the single trace terms [24-26], we obtain

$\langle\mathcal{O}\rangle_{\text {mABJM }}=\operatorname{Tr}\left(Y_{0}^{A} Y_{A 0}^{\dagger}\right)+\frac{1}{N}-$ corrections.

Therefore, in large $N$ limit, the leading term for the vev of the gauge invariant operators are determined by the classical vacuum solutions in (4.48). In particular, the vev of the CPO of conformal dimension one in (4.52) is

$$
\begin{aligned}
\left\langle\mathcal{O}^{(\Delta=1)}\right\rangle_{\mathrm{mABJ}} & =\left.\frac{1}{4} \operatorname{Tr}\left(Z^{a} Z_{a}^{\dagger}-W^{\dagger a} W_{a}\right)\right|_{Z^{a}, W^{\dagger a}=Z_{0}^{a}, W_{0}^{\dagger a}}+\cdots \\
& =\frac{k \mu_{0}}{2 \pi} \sum_{n=0}^{\infty} n(n+1)\left(N_{n}-N_{n}^{\prime}\right)+\cdots
\end{aligned}
$$

where from here on the ellipses denotes the $\frac{1}{N}$-corrections, and $N_{n}$ and $N_{n}^{\prime}$ satisfy the conditions in (4.50). It should be noted that, if one is tempted to make a comparison between the field theory result and the corresponding results from holographic calculations, at finite $N$, one needs to include all the $\frac{1}{N}$-corrections. Otherwise the agreement between the two sides is only expected in the large $N$ limit. See below. Using the one-to-one map in (4.51), we rewrite the vev in (4.57) in terms of the discrete torsions of the dual LLM geometry,

$$
\left\langle\mathcal{O}^{(\Delta=1)}\right\rangle_{\mathrm{mABJ}}=\frac{k \mu_{0}}{2 \pi} \sum_{n=0}^{\infty} n(n+1)\left(\ell_{n}^{(k)}-\ell_{n}^{\prime(k)}\right)+\cdots
$$

\section{Holographic vacuum expectation values}

In Sect. 3, we have constructed the equations of motion (3.35) for 4-dimensional graviton in which the graviton is coupled to the two scalar fields $T$ and $\Psi$. The scalar field $\Psi$ is dual to the CPO in (4.52), whereas $T$ is dual to a gauge-invariant operator of conformal dimension two and does not play a role in the holographic calculation of the vevs of $\mathcal{O}^{(\Delta=1)}$. Thus, let us neglect the scalar field $T$ and consider the action for 4-dimensional graviton and the scalar field $\Psi$,

$$
\begin{aligned}
S= & \frac{1}{16 \pi G_{4}} \int d^{4} x \sqrt{-g}(\hat{R}-2 \Lambda) \\
& -\frac{A_{\Psi}}{2} \int d^{4} x \sqrt{-g}\left(\partial_{\mu} \Psi \partial^{\mu} \Psi+M_{\Psi}^{2} \Psi^{2}\right) .
\end{aligned}
$$

This action reproduces the equation of motion in (3.35), if the multiplicative factor is set to $\sqrt{8 \pi G_{4} A_{\Psi}}=\frac{1}{48}$. By the field redefinition $\tilde{\Psi}=\sqrt{16 \pi G_{4} A_{\Psi}} \Psi$, the action in (5.59) takes canonical form,

$$
\begin{aligned}
S= & \frac{N^{2}}{3 \sqrt{2 \pi^{2} \lambda} L^{2}} \int d^{4} x \sqrt{-g} \\
& \times\left[\hat{R}-2 \Lambda-\frac{1}{2}\left(\partial_{\mu} \tilde{\Psi} \partial^{\mu} \tilde{\Psi}+M_{\Psi}^{2} \tilde{\Psi}^{2}\right)\right],
\end{aligned}
$$

where the 4-dimensional gravitational coupling $G_{4}$ is expressed as

$$
\frac{1}{16 \pi G_{4}}=\frac{N^{2}}{3 \sqrt{2 \pi^{2} \lambda} L^{2}}
$$

with the ' $t$ Hooft coupling constant $\lambda=N / k$ in the ABJM theory [1]. The solution for the rescaled field can be read from the asymptotic expansion of the LLM geometries in (3.45),

$\tilde{\Psi}=-\frac{1}{\sqrt{2}} \beta_{3} \mu_{0} z+\mathcal{O}\left(\mu_{0}^{3}\right)$.

In odd dimensions, the Gubser, Klebanov, PolyakovWitten (GKP-W) relation $[4,5]$ states that the vev of a gauge invariant operator with conformal dimension $\Delta$ is obtained via the holographic renormalization procedure [27-36] as

$\left\langle\mathcal{O}^{(\Delta)}\right\rangle_{\mathrm{HR}}=\frac{N^{2}}{3 \sqrt{2 \pi^{2} \lambda}}(2 \Delta-d) \tilde{\psi}_{\Delta}$,

where $\tilde{\psi}_{\Delta}$ is the coefficient of $z^{\Delta}$ term in the asymptotic expansion of the scalar field $\tilde{\Psi}$. Plugging (3.46) and (5.62) into (5.63) results in the holographic vev of the $\mathrm{CPO}$ of conformal dimension one [10,11]

$\left\langle\mathcal{O}^{(\Delta=1)}\right\rangle_{\mathrm{HR}}=\frac{N^{2} \beta_{3} \mu_{0}}{6 \pi \sqrt{\lambda}}=\frac{N^{3 / 2} \sqrt{k} A_{3} \mu_{0}}{2 \pi A_{2}^{3 / 2}}$.

Subsequently, we employ the gauge/gravity duality dictionary and discuss the effect of the asymptotic discrete torsion $l$ on the vevs.

\subsection{Comparison of gravity and field theory results}

In order to figure out the role of the asymptotic discrete torsion $l$ in the holographic calculation of the vev, we compare the field theory result in (4.58) to the holographic result in (5.64). Since it is not clear how to evaluate the infinite summations in (2.25) and (4.58), it is difficult to make the comparison with full generality. We explicitly show our results by using the example of LLM geometries represented by a rectangular Young diagram in Fig. 2 and then discuss the general picture. 

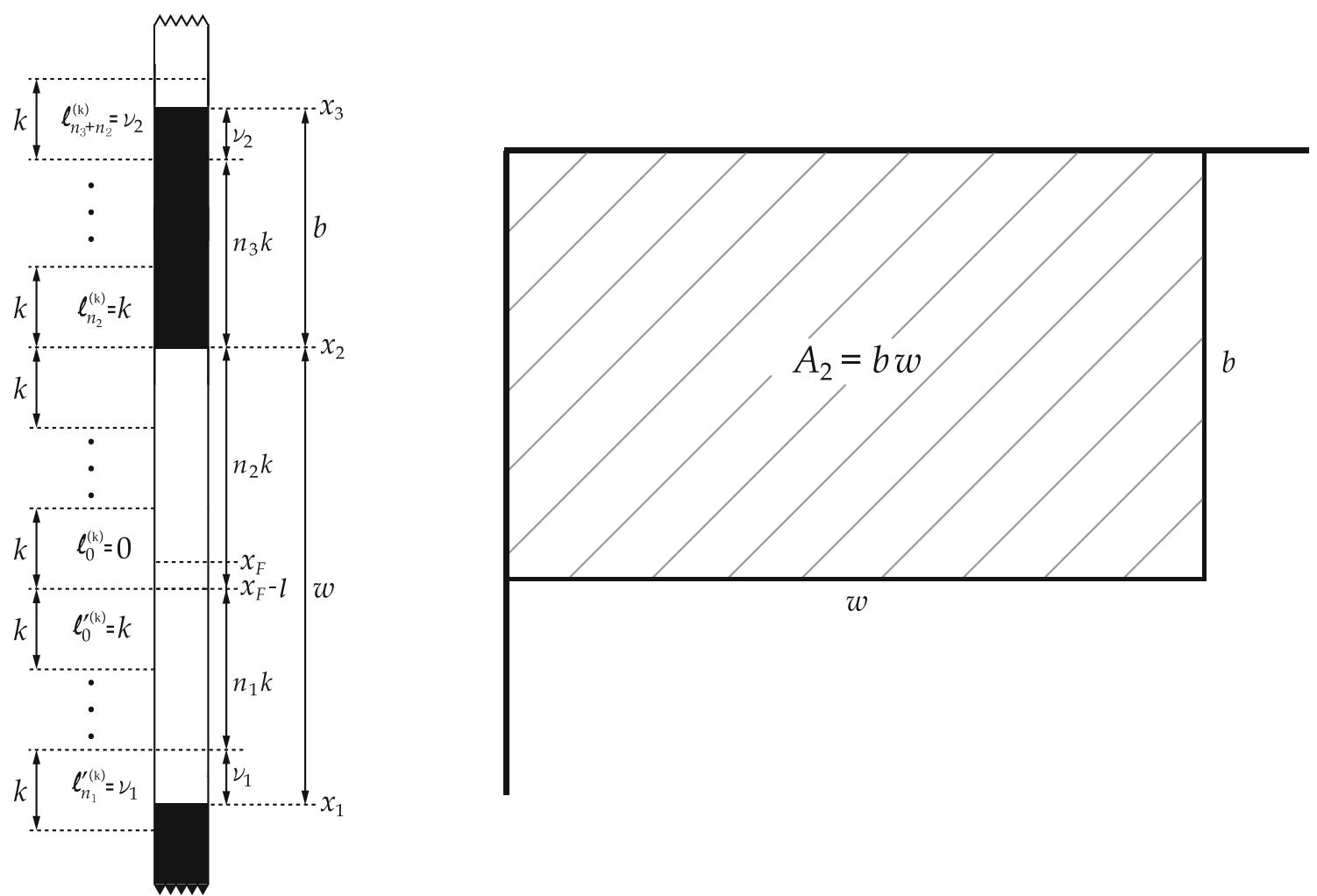

Fig. 2 The droplet and the Young diagram representation of LLM geometries with single finite size black/white region

From the droplet picture in Fig. 2, we read the values for the discrete torsions in the following table,

\begin{tabular}{lr}
\hline$\left\{\ell_{n}^{(k)}, \ell_{n}^{\prime(k)}\right\}$ & range of $n$ \\
\hline \hline$\ell_{n}^{(k)}=0$, & $0 \leq n \leq n_{2}-1$ \\
\hline$\ell_{n}^{(k)}=k$, & $n_{2} \leq n \leq n_{2}+n_{3}-1$ \\
\hline$\ell_{n}^{(k)}=v_{2}$, & $n=n_{2}+n_{3}$ \\
\hline$\ell_{n}^{(k)}=0$, & $n \geq n_{2}+n_{3}+1$ \\
\hline$\ell_{n}^{\prime(k)}=k$, & $0 \leq n \leq n_{1}-1$ \\
\hline$\ell_{n}^{\prime(k)}=v_{1}$, & $n=n_{1}$ \\
\hline$\ell_{n}^{\prime(k)}=0$, & $n \geq n_{1}+1$ \\
\hline
\end{tabular}

where $v_{1}$ and $v_{2}$ are non-negative integers smaller than $k$. Using the relations $w=\left(n_{1}+n_{2}\right) k+v_{1}$ and $b=n_{3} k+$ $\nu_{2}$, together with the level-matching condition in (2.15), we obtain

$n_{1}=\frac{b-l-v_{1}}{k}, \quad n_{2}=\frac{w-b+l}{k}, \quad n_{3}=\frac{b-v_{2}}{k}$,

where $b$ and $w$ are the lengths of the finite black and white regions, respectively. For later convenience, we set $w=\delta b$ without loss of generality for some finite and positive $\delta$ and rewrite $n_{2}$ as

$n_{2}=\frac{(\delta-1) b+l}{k}$.
In [37], it was argued that the LLM geometries are highly curved if they are represented by Young diagrams that contain some short sides. A way to avoid such highly curved geometries is to assume that both $b$ and $w$ are of the order of $\sqrt{N}$. Actually, the relation between $(b, w)$ and $N$ is obtained by plugging the values of the discrete torsions (5.65) into (2.16),

$b=\sqrt{\frac{v_{1}^{2}+v_{2}^{2}-l^{2}+\left(2 N-v_{1}-v_{2}+l\right) k}{2 \delta}}$.

The vev of the $\mathrm{CPO}$ of conformal dimension $\Delta=1$ is evaluated in the mABJ theory by plugging the discrete torsions (5.65) into (4.58), accompanied by the expansion in large $N$ limit,

$$
\begin{aligned}
& \left\langle\mathcal{O}^{(\Delta=1)}\right\rangle_{\mathrm{mABJ}}=\frac{\mu_{0} N^{3 / 2}}{2 \pi}\left(\sqrt{\frac{k}{\delta}}(\delta-1)\right. \\
& +\frac{2 l}{\sqrt{N}}+\frac{3 l(k-l)(\delta-1)}{4 \sqrt{\delta k} N} \\
& -\frac{1}{4 \sqrt{\delta k} N}\left[v_{1}\left(k-v_{1}\right)(1+3 \delta)-v_{2}\left(k-v_{2}\right)(3+\delta)\right] \\
& \left.+\mathcal{O}\left(N^{-3 / 2}\right)\right) \\
& +\cdots,
\end{aligned}
$$


where we truncated the expansion at $\frac{1}{N}$ order for simplicity. Similarly, the holographic vev is obtained by plugging the discrete torsions (5.65) into (5.64). However, now when we expand in the large $N$ limit, the subleading terms are cancelled and only the leading term survive ${ }^{5}$

$\left\langle\mathcal{O}^{(\Delta=1)}\right\rangle_{\mathrm{HR}}=\frac{\mu_{0} N^{3 / 2}}{2 \pi}\left[\sqrt{\frac{k}{\delta}}(\delta-1)\right]$.

Comparing the field theory and the holographic vevs, we confirm an agreement, if the quantum corrections on the field theory side in (5.69) are given by

$$
\begin{aligned}
& -\frac{\mu_{0} N^{3 / 2}}{2 \pi}\left(\frac{2 l}{\sqrt{N}}+\frac{3 l(k-l)(\delta-1)}{4 \sqrt{\delta k} N}\right. \\
& -\frac{1}{4 \sqrt{\delta k} N}\left[v_{1}\left(k-v_{1}\right)(1+3 \delta)-v_{2}\left(k-v_{2}\right)(3+\delta)\right] \\
& \left.+\mathcal{O}\left(N^{-3 / 2}\right)\right)
\end{aligned}
$$

This result shows that the quantum corrections are of order of $\frac{1}{N}$ when the asymptotic discrete torsion $l=0$ as it was anticipated in $[10,11]$, whereas an additional $\frac{1}{\sqrt{N}}$ order correction appears in the presence of the asymptotic discrete torsion $l$.

We have made similar comparisons for LLM geometries with more complex Young diagrams and the behavior is the same as that of the rectangular Young diagram. For instance, the results for the LLM geometries represented by a Young diagram with four long sides are the following. When we have set the lengths of the sides as $\left(w_{1}=\delta_{1} b, b_{1}=\delta_{2} b, w_{2}=\right.$ $\left.\delta_{3} b, b_{2}=b\right), b$ is determined by (2.16),

$b=\sqrt{\frac{v_{1}^{2}+v_{2}^{2}+v_{3}^{2}-l^{2}+k\left(2 N-v_{1}-v_{2}-v_{3}+l\right)}{2\left(\delta_{1}+\delta_{1} \delta_{2}+\delta_{3}\right)}}$,

where $v_{i}$ 's are non-negative integers smaller than $k$. Then we obtain

\footnotetext{
${ }^{5}$ Following [13], we have also tested the case of CPO of conformal dimension $\Delta=2$ and we found the holographic vev is still $N$ exact and is given by
}

$\left\langle\mathcal{O}^{(\Delta=2)}\right\rangle_{\mathrm{HR}}=-\frac{\mu_{0}^{2} N^{3 / 2}}{6 \pi}\left[\frac{\sqrt{k}}{\delta}(\delta+1)^{2}\right]$.

$$
\begin{aligned}
\left\langle\mathcal{O}^{(\Delta}\right. & =1)\rangle_{\mathrm{mABJ}} \\
= & \frac{\mu_{0} N^{3 / 2}}{2 \pi}\left(\frac{\sqrt{k}\left[\delta_{1}\left(\delta_{2}+1\right)\left(\delta_{1}-\delta_{2}-1\right)+\left(2 \delta_{1}+\delta_{3}-1\right) \delta_{3}\right]}{\left(\delta_{1}+\delta_{1} \delta_{2}+\delta_{3}\right)^{3 / 2}}\right. \\
& +\frac{2 l}{\sqrt{N}} \\
& +\frac{3 l(k-l)\left[\delta_{1}\left(\delta_{2}+1\right)\left(\delta_{1}-\delta_{2}-1\right)+\left(2 \delta_{1}+\delta_{3}-1\right) \delta_{3}\right]}{4 \sqrt{k} N\left(\delta_{1}+\delta_{1} \delta_{2}+\delta_{3}\right)^{3 / 2}} \\
& -\frac{v_{1}\left(k-v_{1}\right)\left[\delta_{1}\left(\delta_{2}+1\right)\left(3 \delta_{1}+\delta_{2}+1\right)+\left(6 \delta_{1}+4 \delta_{2}+3 \delta_{3}+1\right) \delta_{3}\right]}{4 \sqrt{k} N\left(\delta_{1}+\delta_{1} \delta_{2}+\delta_{3}\right)^{3 / 2}} \\
& +\frac{v_{2}\left(k-v_{2}\right)\left[\delta_{1}\left(\delta_{2}+1\right)\left(\delta_{1}+3 \delta_{2}+1\right)-\left(2 \delta_{1}+3 \delta_{3}+1\right) \delta_{3}\right]}{4 \sqrt{k} N\left(\delta_{1}+\delta_{1} \delta_{2}+\delta_{3}\right)^{3 / 2}} \\
& +\frac{v_{3}\left(k-v_{3}\right)\left[\delta_{1}\left(\delta_{2}+1\right)\left(\delta_{1}+3 \delta_{2}+3\right)+\left(4 \delta_{1} \delta_{2}+2 \delta_{1}+\delta_{3}+3\right) \delta_{3}\right]}{4 \sqrt{k} N\left(\delta_{1}+\delta_{1} \delta_{2}+\delta_{3}\right)^{3 / 2}} \\
& \left.+\mathcal{O}\left(N^{-3 / 2}\right)\right)+\cdots,
\end{aligned}
$$

and

$$
\begin{aligned}
& \left\langle\mathcal{O}^{(\Delta=1)}\right\rangle_{\mathrm{HR}} \\
& \quad=\frac{\mu_{0} N^{3 / 2}}{2 \pi} \frac{\sqrt{k}\left[\delta_{1}\left(\delta_{2}+1\right)\left(\delta_{1}-\delta_{2}-1\right)+\left(2 \delta_{1}+\delta_{3}-1\right) \delta_{3}\right]}{\left(\delta_{1}+\delta_{1} \delta_{2}+\delta_{3}\right)^{3 / 2}} .
\end{aligned}
$$

The quantum corrections in (5.74) are then given by

$$
\begin{aligned}
- & \frac{\mu_{0} N^{3 / 2}}{2 \pi}\left(\frac{2 l}{\sqrt{N}}+\frac{3 l(k-l)\left[\delta_{1}\left(\delta_{2}+1\right)\left(\delta_{1}-\delta_{2}-1\right)+\left(2 \delta_{1}+\delta_{3}-1\right) \delta_{3}\right]}{4 \sqrt{k} N\left(\delta_{1}+\delta_{1} \delta_{2}+\delta_{3}\right)^{3 / 2}}\right. \\
- & \frac{\nu_{1}\left(k-v_{1}\right)\left[\delta_{1}\left(\delta_{2}+1\right)\left(3 \delta_{1}+\delta_{2}+1\right)+\left(6 \delta_{1}+4 \delta_{2}+3 \delta_{3}+1\right) \delta_{3}\right]}{4 \sqrt{k} N\left(\delta_{1}+\delta_{1} \delta_{2}+\delta_{3}\right)^{3 / 2}} \\
+ & \frac{\nu_{2}\left(k-v_{2}\right)\left[\delta_{1}\left(\delta_{2}+1\right)\left(\delta_{1}+3 \delta_{2}+1\right)-\left(2 \delta_{1}+3 \delta_{3}+1\right) \delta_{3}\right]}{4 \sqrt{k} N\left(\delta_{1}+\delta_{1} \delta_{2}+\delta_{3}\right)^{3 / 2}} \\
+ & \frac{\nu_{3}\left(k-v_{3}\right)\left[\delta_{1}\left(\delta_{2}+1\right)\left(\delta_{1}+3 \delta_{2}+3\right)+\left(4 \delta_{1} \delta_{2}+2 \delta_{1}+\delta_{3}+3\right) \delta_{3}\right]}{4 \sqrt{k} N\left(\delta_{1}+\delta_{1} \delta_{2}+\delta_{3}\right)^{3 / 2}} \\
+ & \left.\mathcal{O}\left(N^{-3 / 2}\right)\right) .
\end{aligned}
$$

Absence of the subleading terms in (5.71), (5.70), and (5.75) shows that $\left\langle\mathcal{O}^{(\Delta)}\right\rangle_{\mathrm{HR}}$ is an exact result in 11dimensional tree-level supergravity. In $[10,11]$, we have verified that there is an exact agreement between the classical field theory result and the holographic result for the special case $k=1$. In such special case, our result here replicates those results, because all $v_{i}$ 's, which are non-negative integers smaller than $k$, are zero. Therefore, the quantum corrections in (5.72) and (5.76) are vanishing in this special case and the classical field theory results has an exact agreement with the holographic results. However, in the general case of $k>1$ and $0 \leq l<k$, such exact agreement is achieved only when we include all the quantum corrections in the field theory calculations. 
$5.2 \frac{1}{\sqrt{N}}$ correction from the discrete torsion

In order to reveal the significance of $l$ in the holographic results, it is necessary to express (5.71) in terms of the parameters of the theory, that can be achieved by eliminating $\delta$ from (5.71) by using (5.67) and (5.68). Then we obtain

$$
\left\langle\mathcal{O}^{(\Delta=1)}\right\rangle_{\mathrm{HR}}=\frac{\mu_{0} N^{3 / 2}}{2 \pi}\left[\frac{\sqrt{2 k}\left(n_{2} k-l\right)}{\sqrt{v_{1}^{2}+v_{2}^{2}-l^{2}+\left(2 N-v_{1}-v_{2}+l\right) k}}\right] .
$$

If $n_{2} k \ll \sqrt{N}$, the leading term is of the same order as the $l$-dependent term, and thus both are vanishing in the large $N$ limit. This case corresponds to the LLM geometries represented by Young diagrams that are almost a square and the vev is vanishing in the case. On the other hand, if the Young diagrams are considered far from being a square, $n_{2} k=\alpha \sqrt{N}$ for some finite value $\alpha$ and thus the vev does not vanish and becomes

$$
\left\langle\mathcal{O}^{(\Delta=1)}\right\rangle_{\mathrm{HR}}=\frac{\mu_{0} N^{3 / 2}}{2 \pi}\left[\alpha-\frac{l}{\sqrt{N}}+\mathcal{O}\left(\frac{1}{N}\right)\right] .
$$

It means the asymptotic discrete torsion $l$ affects the holographic vev only in the subleading order.

\section{Conclusion}

We have investigated the duality between the 3-dimensional $\mathrm{mABJ}$ theory and the 11-dimensional supergravity on LLM geometries with asymptotic discrete torsion $l$. We have calculated the vev of the CPO with conformal dimension $\Delta=1$ from the classical vacuum solutions of the mABJ theory and from the asymptotic limit of the LLM geometries with $\mathbb{Z}_{k}$ orbifold. In our previous works [10,11], we have verified that, in the special case of $k=1$, there is an exact agreement between the result obtained from the field theory classical vacuum solutions and from the holographic methods, whereas for LLM geometries with orbifold singularity, such agreement is reached only in the large $N$ limit. In this paper, we have argued that the mismatch between the two results for $k>1$ is attributed to the missing quantum corrections in the field theory calculations.

We have showed that the holographic vev can be expressed by infinite summations involving the discrete torsions $\left\{\ell_{n}^{(k)}\right.$, $\left.\ell_{n}^{\prime(k)}\right\}$, which are fixed by the details of the droplet pictures representing the LLM geometries. The vevs for CPOs with $\Delta=1,2$ depend on the shapes of droplets except for the overall $N^{3 / 2}$-factor. Relying on the examples of LLM geometries represented by some specific droplet pictures, we have shown that these infinite summations can be evaluated and the holographic vev is exactly determined. However, the vev obtained from the field theory classical vacuum solutions, in addition to the leading order term which agrees with the holographic $v e v$, it contains $\frac{l}{\sqrt{N}}$ corrections when $k>1$ and $l \neq 0$. Since the gauge/gravity duality requires equality of the holographic vev and the one determined from the quantum field theory, including quantum corrections, we have argued that the $\frac{l}{\sqrt{N}}$-corrections from classical vacuum solutions should be matched by the quantum corrections. We have also tested our holographic calculations for the CPO of conformal dimension $\Delta=2$ and the result is still $N$ exact. We have also showed that the holographic vevs have $\frac{l}{\sqrt{N}}$ corrections in the large $N$-limit.

The holographic methods involve the asymptotic expansion of the LLM geometries. The asymptotic limit of the LLM geometries with the $\mathbb{Z}_{k}$ orbifold is the $\mathrm{AdS}_{4} \times S^{7} / \mathbb{Z}_{k}$ geometry with the radius $\left(L=\left(32 \pi^{2} A_{2}\right)^{1 / 6} l_{\mathrm{P}}\right)$ of the $S^{7}$ determined by the details of the droplet picture of the original LLM geometry. In the $k=1$ case the radius depends only on $N$ and the asymptotic geometry does not depend on the details of the droplet picture of the LLM geometries. Therefore, in the absence of the orbifolding, all LLM geometries with the same value of $N$ have the same asymptotic geometry, whereas such degeneracy is removed for the LLM geometries with the orbifold singularity.

Acknowledgements This work was supported by the National Research Foundation of Korea (NRF) Grant with Grant number NRF2018R1D1A1B07048061 (D.J.), NRF-2019R1F1A1056815 (Y.K.), NRF-2017R1D1A1A09000951, NRF-2019R1F1A1059220, NRF2019R1A6A1A10073079 (O.K.), and NRF-2017R1D1A1B03032523 (D.T.).

Data Availability Statement This manuscript has no associated data or the data will not be deposited. [Authors' comment: This is a theoretical study and no experimental data has been listed.]

Open Access This article is licensed under a Creative Commons Attribution 4.0 International License, which permits use, sharing, adaptation, distribution and reproduction in any medium or format, as long as you give appropriate credit to the original author(s) and the source, provide a link to the Creative Commons licence, and indicate if changes were made. The images or other third party material in this article are included in the article's Creative Commons licence, unless indicated otherwise in a credit line to the material. If material is not included in the article's Creative Commons licence and your intended use is not permitted by statutory regulation or exceeds the permitted use, you will need to obtain permission directly from the copyright holder. To view a copy of this licence, visit http://creativecomm ons.org/licenses/by/4.0/.

Funded by $\mathrm{SCOAP}^{3}$.

\section{References}

1. O. Aharony, O. Bergman, D.L. Jafferis, J. Maldacena, $N=6$ superconformal Chern-Simons-matter theories, M2-branes and their gravity duals. JHEP 0810, 091 (2008). arXiv:0806.1218 [hep-th] 
2. J.M. Maldacena, The Large N limit of superconformal field theories and Int. J. Theor. Phys. 38, 1113 (1999)

3. J.M. Maldacena, Adv. Theor. Math. Phys. 2, 231 (1998). arXiv:hep-th/9711200

4. S.S. Gubser, I.R. Klebanov, A.M. Polyakov, Gauge theory correlators from noncritical string theory. Phys. Lett. B 428, 105 (1998). arXiv:hep-th/9802109

5. E. Witten, Anti-de Sitter space and holography. Adv. Theor. Math. Phys. 2, 253 (1998). arXiv:hep-th/9802150

6. K. Hosomichi, K.M. Lee, S. Lee, S. Lee, J. Park, N = 5,6 superconformal Chern-Simons theories and M2-branes on orbifolds. JHEP 0809, 002 (2008). arXiv:0806.4977 [hep-th]

7. J. Gomis, D. Rodriguez-Gomez, M. Van Raamsdonk, H. Verlinde, A massive study of M2-brane proposals. JHEP 0809, 113 (2008). arXiv:0807.1074 [hep-th]

8. H. Lin, O. Lunin, J.M. Maldacena, Bubbling AdS space and 1/2 BPS geometries. JHEP 0410, 025 (2004). arXiv:hep-th/0409174

9. S. Cheon, H.C. Kim, S. Kim, Holography of mass-deformed M2branes. arXiv:1101.1101 [hep-th]

10. D. Jang, Y. Kim, O.K. Kwon, D.D. Tolla, Exact holography of the mass-deformed M2-brane theory. Eur. Phys. J. C 77(5), 342 (2017). arXiv: 1610.01490

11. D. Jang, Y. Kim, O.K. Kwon, D.D. Tolla, Mass-deformed ABJM theory and LLM geometries: exact holography. JHEP 1704, 104 (2017). arXiv:1612.05066 [hep-th]

12. O. K. Kwon, D. Jang, Y. Kim, D. D. Tolla, "Holography of Massive M2-brane Theory: Non-linear Extension,” Eur. Phys. J. C 78, no. 10, 856 (2018) arXiv:1803.10660 [hep-th]

13. O.K. Kwon, D. Jang, Y. Kim, D.D. Tolla, Gravity from entanglement and RG flow in a top-down approach. JHEP 1805, 009 (2018). arXiv:1712.09101 [hep-th]

14. S. Ryu, T. Takayanagi, Holographic derivation of entanglement entropy from AdS/CFT. Phys. Rev. Lett. 96, 181602 (2006). arXiv:hep-th/0603001

15. T. Faulkner, Bulk emergence and the RG flow of entanglement entropy. JHEP 1505, 033 (2015). arXiv:1412.5648 [hep-th]

16. K.K. Kim, O.K. Kwon, C. Park, H. Shin, Renormalized entanglement entropy flow in mass-deformed ABJM theory. Phys. Rev. D 90(4), 046006 (2014). arXiv:1404.1044

17. K.K. Kim, O.K. Kwon, C. Park, H. Shin, Holographic entanglement entropy of mass-deformed Aharony-Bergman-JafferisMaldacena theory. Phys. Rev. D 90(12), 126003 (2014). arXiv:1407.6511 [hep-th]

18. C. Kim, K.K. Kim, O.K. Kwon, Holographic entanglement entropy of anisotropic minimal surfaces in LLM geometries. Phys. Lett. B 759, 395 (2016). arXiv:1605.00849 [hep-th]

19. O. Aharony, O. Bergman, D.L. Jafferis, Fractional M2-branes. JHEP 0811, 043 (2008). arXiv:0807.4924 [hep-th]
20. R. Auzzi, S.P. Kumar, Non-Abelian vortices at weak and strong coupling in mass deformed ABJM theory. JHEP 0910, 071 (2009). arXiv:0906.2366 [hep-th]

21. K. Skenderis, M. Taylor, Kaluza-Klein holography. JHEP $\mathbf{0 6 0 5}$, 057 (2006). arXiv:hep-th/0603016

22. K. Skenderis, M. Taylor, Holographic Coulomb branch vevs. JHEP 0608, 001 (2006). arXiv:hep-th/0604169

23. H.C. Kim, S. Kim, Supersymmetric vacua of mass-deformed M2brane theory. Nucl. Phys. B 839, 96 (2010). arXiv:1001.3153 [hepth]

24. E. Witten, Multitrace operators, boundary conditions, and AdS / CFT correspondence. arXiv:hep-th/0112258

25. M. Berkooz, A. Sever, A. Shomer, 'Double trace' deformations, boundary conditions and space-time singularities. JHEP 0205, 034 (2002). arXiv:hep-th/0112264

26. S.S. Gubser, I.R. Klebanov, A Universal result on central charges in the presence of double trace deformations. Nucl. Phys. B 656, 23 (2003). arXiv:hep-th/0212138

27. V. Balasubramanian, P. Kraus, A stress tensor for antide Sitter gravity. Commun. Math. Phys. 208, 413 (1999). arXiv:hep-th/9902121

28. S. de Haro, S.N. Solodukhin, K. Skenderis, Holographic reconstruction of space-time and renormalization in the AdS / CFT correspondence. Commun. Math. Phys. 217, 595 (2001). arXiv:hep-th/0002230

29. K. Skenderis, Asymptotically anti-de Sitter space-times and their stress energy tensor. Int. J. Mod. Phys. A 16, 740 (2001). arXiv:hep-th/0010138

30. M. Bianchi, D.Z. Freedman, K. Skenderis, Holographic renormalization. Nucl. Phys. B 631, 159 (2002). arXiv:hep-th/0112119

31. M. Henningson, K. Skenderis, The holographic Weyl anomaly. JHEP 9807, 023 (1998). arXiv:hep-th/9806087

32. J. de Boer, E.P. Verlinde, H.L. Verlinde, On the holographic renormalization group. JHEP 0008, 003 (2000). arXiv:hep-th/9912012

33. P. Kraus, F. Larsen, R. Siebelink, The gravitational action in asymptotically AdS and flat space-times. Nucl. Phys. B 563, 259 (1999). arXiv:hep-th/9906127

34. M. Bianchi, D.Z. Freedman, K. Skenderis, How to go with an RG flow. JHEP 0108, 041 (2001). arXiv:hep-th/0105276

35. D. Martelli, W. Mueck, Holographic renormalization and Ward identities with the Hamilton-Jacobi method. Nucl. Phys. B 654, 248 (2003). arXiv:hep-th/0205061

36. K. Skenderis, Lecture notes on holographic renormalization. Class. Quant. Gravit. 19, 5849 (2002). arXiv:hep-th/0209067

37. Y.H. Hyun, Y. Kim, O.K. Kwon, D.D. Tolla, Abelian projections of the mass-deformed ABJM theory and weakly curved dual geometry. Phys. Rev. D 87(8), 085011 (2013). arXiv:1301.0518 [hep-th] 